\title{
Complementarity problems via common fixed points in vector lattices
}

\author{
Mujahid Abbas ${ }^{1}$, Abdul Rahim Khan ${ }^{2 *}$ and SZ Németh ${ }^{3}$
}

\author{
* Correspondence: arahim@kfupm. \\ edu.sa \\ ${ }^{2}$ Department of Mathematics and \\ Statistics, King Fahd University of \\ Petroleum and Minerals, Dhahran \\ 31261, Saudi Arabia \\ Full list of author information is \\ available at the end of the article
}

\begin{abstract}
Nemeth introduced the notion of order weakly L-Lipschitz mapping and employed this concept to obtain nontrivial solutions of nonlinear complementarity problems. In this article, we shall extend this concept to two mappings and obtain the solution of common fixed point equations and hence coincidence point equations in the framework of vector lattices. We present some examples to show that the solution of nonlinear complementarity problems and implicit complementarity problems can be obtained using these results. We also provide an example of a mapping for which the conclusion of Banach contraction principle fails but admits one of our fixed point results. Our proofs are simple and purely order-theoretic in nature.
\end{abstract}

Keywords: coincidence point equation, weak order contractive condition, order convergence, vector lattice, mutually dominating maps

\section{Introduction and preliminaries}

Let $E$ be a vector space. A closed convex set $K$ in $E$ is called closed convex cone, if for any $\lambda>0$ and $x \in K, \lambda x \in K$. A convex cone $K$ is called pointed if $K \cap(-K)=\{0\}$. From now on we shall call a closed convex pointed cone simply cone.

A relation $\rho$ on $E$ is called: $(i)$ reflexive if $x \rho x$ for all $x \in E$. (ii) transitive if $x \rho y$ and $y \rho z$ imply $x \rho z$, where $x, y, z \in E$. (iii) antisymmetric if $x \rho y$ and $y \rho x$ imply $x=y$ for $x, y$ $\in E$. (iv) preorder if it is reflexive and transitive. (v) translation invariant if $x \rho y$ implies $(x+z) \rho(y+z)$ for any $z \in E$ whenever $x, y \in E$. (vi) scale invariant if $x \rho y$ implies $(\lambda x)$ $\rho(\lambda y)$ for any $\lambda>0, x, y \in E$.

A preorder is called a partial order if it is antisymmetric. Let $K$ be a cone in $E$. Denote $x \leq y$ if $y-x \in K$. Then, $\leq$ defines a partial order on $E$ called the order induced by $K$. Conversely, if $\leq$ is a partial order on $E$, then $E$ is called ordered vector space and the set $K=\{x \in E: 0 \leq x\}$ is a cone called the positive cone of $E$. In this case it is easy to see that $x<y$ if and only if $y-x \in E$. The ordered vector space $(E, \leq)$ is called a vector lattice if for every $x, y \in E$, there exist $x \wedge y:=\inf \{x, y\}$ and $x \vee y:=\sup \{x, y\}$.

If we denote $x^{+}=0 \vee x, x^{-}=0 \vee(-x)$ and $|x|=x \vee(-x)$, then $x=x^{+}-x^{-}$and $|x|=x^{+}+x^{-}$. Note that $|\alpha x|=|\alpha| \cdot|x|$, for $\alpha \in \mathbb{R}|| x|-| y|| \leq|x-y|$ and $|x+y| \leq|x|+|y|$ for $x, y \in E$.

Note that if $a \leq h a$ where $a \in K$ and $h \in(0,1)$, then $a=0$.

Definition $1 A$ sequence of vectors $\left\{x_{n}\right\}$ in $E$ is said to: (i) decrease to an element $x \in$ $E$ if $x_{n+1} \leq x_{n}$ for every $n$ in $\mathbb{N}$ (set of natural numbers) and

(C) 2012 Abbas et al; licensee Springer. This is an Open Access article distributed under the terms of the Creative Commons Attribution License (http://creativecommons.org/licenses/by/2.0), which permits unrestricted use, distribution, and reproduction in any medium, provided the original work is properly cited. 
$x=\sup \left\{x_{n}: n \in \mathbb{N}\right\}=\vee_{n \in \mathrm{N}} x_{n}$. We denote it by $x_{n} \downarrow x$. (ii) increase to an element $x \in E$ if $x_{n} \leq x_{n+1}$ for every $n$ in $\mathbb{N}$ and $x=\sup \left\{x_{n}: n \in \mathbb{N}\right\}=\vee_{n \in \mathbb{N}} x_{n}$. We denote it by $x_{n} \uparrow x$.

Definition $2 A$ sequence of vectors $\left\{x_{n}\right\}$ in $E$ is said to be order convergent to $x \in E$ if there exist sequences $\left\{y_{n}\right\}$ and $\left\{z_{n}\right\}$ in E such that $y_{n} \downarrow x, z_{n} \uparrow x$ and

$$
z_{n} \leq x_{n} \leq y_{n} .
$$

We denote this by $x=$ order $-\lim _{n \rightarrow \infty} x_{n}$. If the sequence is order convergent, then its order limit is unique.

Definition $3 A$ sequence of vectors $\left\{x_{n}\right\}$ in $E$ is said to be order Cauchy sequence in $E$ if the sequence $\left\{x_{m}-x_{n}\right\}$ in $K$ is order convergent to 0 .

Definition 4 Let $E$ and $F$ be two vector lattices. A mapping $f: E \rightarrow F$ is called order continuous at $x_{0}$ in $E$ if for any sequence $\left\{x_{n}\right\}$ in E such that $x=$ order $-\lim _{n \rightarrow \infty} x_{n}$, we have $f(x)=$ order $-\lim _{n \rightarrow \infty} f\left(x_{n}\right)$.

Remark 5 If $x_{n} \in K$ for every $n$ in $\mathbb{N}$ and $x=$ order $-\lim _{n \rightarrow \infty} x_{n}$, then $x \in K$. Also, if $x_{n} \in$ $K$ for every $n$ in $\mathbb{N}$ and $\left\{y_{n}\right\}$ is any sequence for which $y_{n^{-}} x_{n} \in K$ with $0=$ order - $\lim _{n \rightarrow \infty} y_{n}$, then $0=$ order $-\lim _{n \rightarrow \infty} x_{n}$.

Definition $6 \mathrm{~A}$ cone $K \subset E$ is called regular if every decreasing sequence of elements in $K$ is convergent.

Definition $7 A$ vector lattice $E$ is complete if there exists $\sup A$ and $\inf A$ for each bounded countable subset $A$ of $E$.

For more details on vector lattices, order convergence, and order continuity, we refer to [1-3] and references mentioned therein.

Definition 8 If $(E, \leq)$ is a vector lattice and $f: E \rightarrow E$ is such that $f(x) \leq f(y)$ whenever $x, y \in E$ and $x \leq y$, then $f$ is said to be nondecreasing.

Definition 9 Let $(E, \leq)$ be a vector lattice. The set $(U F)_{f}=\{x \in E: x-f(x) \in K\}$ is called upper fixed point set of $f,(L F)_{f}=\{x \in E: f(x)-x \in K\}$ is called lower fixed point set of $f$ and $(F)_{f}=\{x \in E: f(x)=x\}$ is called the set of all fixed points of $f$.

Definition 10 Let $(E, \leq)$ be a vector lattice. The self map $f$ on $E$ is called: (i) dominated on $E$ if $(U F)_{f}=E$. (ii) dominating on $E$ if $(L F)_{f}=E$.

Example 11 Let $E=[0,1]$ be endowed with the usual ordering. Let $f: E \rightarrow E$ be defined by $f(x)=x^{\frac{1}{3}}$. Then $(L F)_{f}=E$.

Example 12 Let $E=[0, \infty)$ be endowed with the usual ordering. Define $f: E \rightarrow E$ by

$$
f(x)=\left\{\begin{array}{l}
\sqrt[n]{x} \text { for } x \in[0,1), \\
x^{n} \text { for } x \in[1, \infty),
\end{array}\right.
$$

$n \in \mathbb{N}$. Then $(L F)_{f}=E$.

The condition $(U F)_{f}=E$ occurs very naturally in several practical problems. For example, if $x$ denotes the total quantity of food produced over a certain period of time and $f(x)$ gives the quantity of food consumed over the same period in a certain town, then we must have $f(x) \leq x$. 
Definition 13 Let $(E, \leq)$ be a vector lattice. Two mappings $f, g: E \rightarrow E$ are said to be mutually dominated if $f(x) \in(U F)_{g}$ and $g(x) \in(U F)_{f}$ for all $x \in E$. That is, $f(x) \geq g(f(x))$ and $g(x) \geq f(g(x))$ for all $x$ in $E$.

Definition 14 Let $(E, \leq)$ be a vector lattice. Two mappings $f, g: E \rightarrow E$ are said to be mutually dominating if $f(x) \in(L F)_{g}$ and $g x \in(L F)_{f}$ for all $x \in E$. That is, $f(x) \leq g(f(x))$ and $g(x) \leq f(g(x))$ for all $x$ in $E$.

The following two examples show that there exist discontinuous and mutually dominating mappings which are not nondecreasing mappings.

Example 15 Let $E=\mathbb{R}_{+}$, be endowed with the usual ordering. Let $f, g: E \rightarrow E$ be defined by

$$
f(x)=\left\{\begin{array}{l}
3 x+5 \text { if } x \in[0,2) \\
4 x+\text { if } x \geq 2
\end{array}\right.
$$

and

$$
g(x)=\left\{\begin{array}{l}
x+7 \text { if } x \in[0,2) \\
3 x+2 \text { if } x \geq 2
\end{array}\right.
$$

Note that for $x \in[0,2)$,

$$
f(x)=3 x+5<9 x+17=3(3 x+5)+2=(g \circ f) x
$$

and

$$
g(x)=x+7<4 x+29=(x+7)+1=(f \circ g) x .
$$

Also, for $x \geq 2$,

$$
f(x)=4 x+1<12 x+5=(4 x+1)+2=(g \circ f) x .
$$

and

$$
g(x)=3 x+2<12 x+9=4(3 x+2)+1=(f \circ g) x .
$$

Thus, $f$ and $g$ are mutually dominating maps. However, for $1.5<2$, we have

$$
f(1.5)=3(1.5)+5=9.5 \not \leq 9=4(2)+1=f(2) .
$$

Also,

$$
g(1.5)=1.5+7=9.5 \not \leq 8=3(2)+2=g(2) .
$$

Hence, $f$ and $g$ both are not nondecreasing maps.

Example 16 Let $E=\mathbb{R}_{+}^{2}$, be endowed with the usual ordering in $\mathbb{R}^{2}$. Let $f, g: E \rightarrow E$ be defined by

$$
f\left(x_{1}, x_{2}\right)=\left\{\begin{array}{l}
\left(2 x_{1}+3 x_{2}+8,4 x_{2}+3\right) \text { if }\left(x_{1}, x_{2}\right) \in[0,1) \times[0,1) \\
\left(x_{1}+5 x_{2}, 5 x_{2}+1\right) \text { if }\left(x_{1}, x_{2}\right) \in[1, \infty) \times[1, \infty) \\
(0,0) \text { otherwise }
\end{array}\right.
$$


and

$$
g\left(x_{1}, x_{2}\right)=\left\{\begin{array}{l}
\left(x_{1}-x_{2}+6,2 x_{2}+4\right) \text { if }\left(x_{1}, x_{2}\right) \in[0,1) \times[0,1) \\
\left(2 x_{1}+x_{2}, 3 x_{2}+1\right) \text { if }\left(x_{1}, x_{2}\right) \in[1, \infty) \times[1, \infty) \\
(0,0) \text { otherwise }
\end{array}\right.
$$

Now for $\left(x_{1}, x_{2}\right) \in[0,1) \times[0,1)$,

$$
\begin{aligned}
f\left(x_{1}, x_{2}\right) & =\left(2 x_{1}+3 x_{2}+8,4 x_{2}+3\right) \\
& \leq\left(x_{1}+9 x_{2}+10,10 x_{2}+21\right) \\
& \left.=\left(2\left(2 x_{1}+3 x_{2}+8\right)+\left(4 x_{2}+3\right), 3\left(4 x_{2}+3\right)+1\right)\right)=(g \circ f)\left(x_{1}, x_{2}\right)
\end{aligned}
$$

and

$$
\begin{aligned}
g\left(x_{1}, x_{2}\right) & =\left(x_{1}-x_{2}+6,2 x_{2}+4\right) \\
& \leq\left(x_{1}+9 x_{2}+10,10 x_{2}+21\right) \\
& \left.=\left(\left(x_{1}-x_{2}+6\right)+5\left(2 x_{2}+4\right), 5\left(2 x_{2}+4\right)+1\right)\right)=(f \circ g)\left(x_{1}, x_{2}\right) .
\end{aligned}
$$

Also for $\left(x_{1}, x_{2}\right) \in[1, \infty) \times[1, \infty)$,

$$
\begin{aligned}
f\left(x_{1}, x_{2}\right) & =\left(x_{1}+5 x_{2}, 5 x_{2}+1\right) \\
& \leq\left(2 x_{1}+15 x_{2}+1,9 x_{2}+4\right) \\
& =\left(2\left(x_{1}+5 x_{2}\right)+\left(5 x_{2}+1\right), 3\left(3 x_{2}+1\right)+1\right)=(g \circ f)\left(x_{1}, x_{2}\right)
\end{aligned}
$$

and

$$
\begin{aligned}
g\left(x_{1}, x_{2}\right) & =\left(2 x_{1}+x_{2}, 3 x_{2}+1\right) \\
& \leq\left(2 x_{1}+16 x_{2}+5,15 x_{2}+6\right) \\
& =\left(\left(2 x_{1}+x_{2}\right)+5\left(3 x_{2}+1\right), 5\left(3 x_{2}+1\right)+1\right)=(f \circ g)\left(x_{1}, x_{2}\right) .
\end{aligned}
$$

Thus, $f$ and $g$ are mutually dominating maps but not nondecreasing. However, for $(0.5,1) \leq(1,1)$, we have

$$
\begin{aligned}
f(0.5,1) & =(2(0.5)+3(1)+8,2(1)+4) \\
& =(12,6) \not \leq(6,6)=(1+5(1), 5(1)+1)=f(1,1) .
\end{aligned}
$$

Also

$$
\begin{aligned}
g(0.5,1) & =(0.5-1+6,2(1)+4) \\
& =(5.5,6) \not \leq(3,4)=(2(1)+1,3(1)+1)=g(1,1) .
\end{aligned}
$$

Hence, $f$ and $g$ are not nondecreasing.

The concept of mutually dominated mappings has recently been employed by Hussain et al. [4] to solve the following implicit integral equation

$$
p(t, x(t))=\int_{0}^{1} q(t, s, x(s)) d s, t \in[0,1]
$$

where $x \in L^{p}[0,1], 1<p<\infty$.

If we take $f=g$ in Definition 13, then we have $f(x) \leq f^{2}(x)$ for all $x$ in $E$; in this case, we call $f$ to be a weakly increasing self map. 
Example 17 Let $E=\mathbb{R}^{+}$be endowed with its usual order and $f: E \rightarrow E$ be defined by

$$
f x=\left\{\begin{array}{l}
x^{\frac{1}{2}}, \text { if } x \in[0,1] \\
2, \text { if } x \in(1,2] \\
1, \text { if } x \in(2, \infty)
\end{array}\right.
$$

Note that if $x \in[0,1], f(x)=x^{\frac{1}{2}} \leq x^{\frac{1}{4}}=f^{2}(x)$, when $x \in(1,2], f(x)=2=f^{2}(x)$, and if $x>2, f(x)=1=f^{2}(x)$. Thus $f(x) \leq f^{2}(x)$ for all $x \in E$, so $f$ is weakly increasing. However, $f$ is not increasing because $2<3$ but $f(2)=2>1=f(3)$.

Definition 18 [5] Let $(E, \leq)$ be a vector lattice and $K$ be its positive cone. A monotone increasing mapping $\phi: K \rightarrow K$ is called comparison operator if $\lim _{n \rightarrow \infty} \phi^{n}(t)=0$ for each $t$ in $K$.

Definition 19 Let $(E, \leq)$ be a vector lattice and $\left\{x_{n}\right\}$ be a sequence in $E$. If order$\lim _{n} \sum_{j=1}^{n} x_{j}$ exists, then we say that the series $\sum_{n=1}^{\infty} x_{n}$ is order-convergent.

Definition 20 [5]Let $(E, \leq)$ be a vector lattice and $K$ be its positive cone. A monotone increasing mapping $\phi: K \rightarrow K$ is called (c)-comparison operator if there exists an order convergent series $\sum_{n=1}^{\infty} x_{n}$ in $K$ and a real number $\alpha \in[0,1)$ such that $\phi^{k+1}(t) \leq \alpha \phi^{k}(t)+$ $x_{k}$ for each $t$ in $K$.

Theorem $21[5]$ Let $(E, \leq)$ be a vector lattice, $K$ be its positive cone and $\sum_{n=1}^{\infty} y_{n}$ be a series of positive terms in E. If there exists an order convergent series $\sum_{n=1}^{\infty} x_{n}$ in $K$ and a real number $\alpha \in[0,1)$ such that $y_{n+1} \leq \alpha y_{n}+x_{n}$, then the series $\sum_{n=1}^{\infty} y_{n}$ is order convergent.

For more details on (c)-comparison operators, we refer to [5] and references mentioned therein.

A fixed point problem is to find some $x$ in $E$ such that $f(x)=x$ and we denote it by $F P(f, E)$. Let $f, g: E \rightarrow E$. A common fixed point problem is to find some $x$ in $E$ such that $x=f(x)=g(x)$ and we denote it by $C F P(f, g, E)$. The equation $f(x)=g(x)(f(x)=g$ $(x)=x)$ is called coincidence point equation (resp. common fixed point equation).

Definition 22 If $H$ is a real Hilbert space with the duality product $\langle\cdot, \cdot\rangle, K \subset H$ a cone, $K^{*}=\{y \in H:\langle x, y\rangle \geq 0$ for all $x \in K\}$ the dual of $K$, and $f: K \rightarrow H$ a mapping, then the nonlinear complementarity problem defined by $f$ and $K$ is to find an $x^{*} \in K$ such that $f\left(x^{*}\right) \in K^{*}$ and $\left\langle x^{*}, f\left(x^{*}\right)\right\rangle=0$.

Complementarity problems are used to model several problems of economics, physics, engineering, and optimization theory. It is known that $x^{*}$ is a solution of the nonlinear complementarity problem defined by $K$ and $f$ if and only if $x^{*}$ is a fixed point of the mapping

$$
F(x)=P_{K}(x-f(x))
$$

where $x \in H$ and $P_{K}$ is the projection mapping of $H$ onto $K$. The nonlinear complementarity problem defined by $K$ and $f$ will be denoted by $N C P(f, K)$. 
Definition 23 For $f, g: K \rightarrow H$, the implicit complementarity problem is to find $x^{*}$ in $K$ such that $g\left(x^{*}\right) \in K, f\left(x^{*}\right) \in K^{*}$ and

$$
\left\langle f\left(x^{*}\right), g\left(x^{*}\right)\right\rangle=0 .
$$

This problem will be denoted by $I C P(f, g, K)$.

It is known that $x^{*}$ is a solution of $\operatorname{ICP}(f, g, K)$ if it is a solution of the coincidence equation

$$
g(x)=P_{K}(g(x)-f(x))
$$

(see Proposition 5 in [6]). The implicit complementarity problem was defined in connection with stochastic optimal control $[7,8]$. If we take $g=I$ (i.e., the inclusion mapping), then $I C P(f, g, K)$ becomes $N C P(f, K)$.

The existence of solutions of fixed point problems in ordered metric spaces has been investigated by Ran and Reurings [9] and Nieto and Lopez [10]. Recently, many mathematicians have considered fixed point, common fixed point, and coincidence point equations in partially ordered metrics spaces (see, e.g., [11-14]). Survey of fixed point theory reveals that most of the results rely on metric structure of the underlying space. Recently, Abbas and Khan (unpublished work) used the notion of modulus of a vector (as used by Berinde [5]) to study the order convergence of Picard and Jungck-type iteration to fixed points and common fixed points of mappings on a $\sigma$-complete vector lattice. Note that the modulus of a vector shares some properties of the norm of a vector. Nemeth [15] introduced weakly Lipschitz mappings in order to obtain a nontrivial solution of nonlinear complementarity problems. Motivated by the work of Nemeth [15], we initiate the study of fixed points and common fixed points of weakly order contractive mappings in the framework of vector lattices. In the existing literature, contractive conditions are imposed on mappings to obtain fixed points and common fixed points. It is remarked that the notion of weakly order contractive mappings purely relies on order structure. To the best of the authors' knowledge, our results are new and rely on order structure of the ambient space and hopefully will open a new avenue of research in this area.

\section{Main results}

The authors in $[9,10,12,14]$ have extensively employed properties of metric on a partially ordered set to prove fixed point results. We establish the existence of common fixed points for weakly order contractive mappings by using only order-theoretic properties as follows:

Theorem 24 Let $(E, \leq)$ be a complete vector lattice and $K$ its positive cone. Let $f, g: E$ $\rightarrow E$ be two mutually dominated mappings with respect to $\leq$ and

$$
f(x)-g(y) \leq \phi(x-y)
$$

provided $x-y \in K$ and $\phi: K \rightarrow K$ is (c) - comparison operator. Then, the common fixed point problem $\operatorname{CFP}(f, g, E)$ has a solution provided that $f$ is order continuous and $g$ is a dominated map.

Proof. Suppose that $x_{0}$ is an arbitrary point of $E$ and $\left\{x_{n}\right\}$ a sequence in $E$ such that $x_{2 n+1}=f\left(x_{2 n}\right)$ and $x_{2 n+2}=g\left(x_{2 n+1}\right)$ for all $n \geq 0$. Since $f$ and $g$ are mutually dominated mappings, therefore $x_{1}=f\left(x_{0}\right) \geq g\left(f\left(x_{0}\right)\right)=g\left(x_{1}\right)=x_{2}=g\left(x_{1}\right) \geq f\left(g\left(x_{1}\right)\right)=f\left(x_{2}\right)=x_{3}$. 
Continuing this process, we have $x_{1} \geq x_{2} \geq \ldots \geq x_{n} \geq x_{n+1} \geq \ldots$. That is, the sequence $\left\{x_{n}\right\}$ is decreasing. Therefore,

$$
x_{2 n+1}-x_{2 n+2}=f\left(x_{2 n}\right)-g\left(x_{2 n+1}\right) \leq \phi\left(x_{2 n}-x_{2 n+1}\right)
$$

for all $n \geq 1$ and consequently

$$
\begin{aligned}
x_{2 n+1}-x_{2 n+2} & \leq \phi\left(x_{2 n}-x_{2 n+1}\right) \leq \phi^{2}\left(x_{2 n-1}-x_{2 n}\right) \\
& \leq \cdots \leq \phi^{2 n}\left(x_{1} x_{2}\right) .
\end{aligned}
$$

for all $n \in \mathbb{N}$, and so we have

$$
\begin{aligned}
x_{n}-x_{n+p} & \leq x_{n}-x_{n+1}+x_{n+1}-x_{n+2}+\cdots+x_{n+p-1}-x_{n+p} \\
& \leq \phi^{n}\left(x_{1}-x_{2}\right)+\phi^{n+1}\left(x_{1}-x_{2}\right)+\cdots+\phi^{n+p-1}\left(x_{1}-x_{2}\right) \\
& \leq \sum_{k=n}^{n+p-1} \phi^{k}\left(x_{1}-x_{2}\right) .
\end{aligned}
$$

Now since $\phi$ is $(c)$-comparison operator, using Theorem 21 (see also the proof of Theorem 2 in [5]), we obtain that $\left\{x_{n}\right\}$ is an order-Cauchy sequence in $E$. Since $E$ is complete, there exists an element $x^{*} \in E$ such that $x^{*}=\operatorname{order}-\lim _{n \rightarrow \infty} x_{n}$. As $f$ is order continuous we have that $f\left(x^{*}\right)=x^{*}$ (fixed point of $f$ ). Since $x^{*} \leq x^{*}$, we obtain

$$
f\left(x^{*}\right)-g\left(x^{*}\right) \leq \phi\left(x^{*}-x^{*}\right)
$$

which implies that $x^{*} \in(L F)_{g}$. Since $g$ is a dominated map, $x^{*}-g\left(x^{*}\right) \geq 0$ implies that $x^{*} \in(U F)_{g}$. So, $C F P(f, g, E)$ has a solution.

The above theorem for mutually dominating mappings can also be established with some obvious slight modifications.

Theorem 25 Let $(E, \leq)$ be a vector lattice and $K$ a regular cone in $E$. Suppose that $f, g$ $: K \rightarrow K$ are two mutually dominated mappings with respect to the order induced by $K$ and

$$
f(x)-g(y) \leq \phi(x-y)
$$

for all $x, y \in K$ with $x-y \in K$ and $\phi: K \rightarrow K$ is (c)-comparison operator. Then, the common fixed point problem $\operatorname{CFP}(f, g, K)$ has a solution provided that $f$ is continuous and $g$ is a dominated map.

Proof. Following arguments similar to those given in the proof of Theorem 24, we obtain a bounded decreasing sequence $\left\{x_{n}\right\}$ in $K$. Since $K$ is regular, therefore there exists some $x^{*}$ in $K$ such that $x^{*}=\lim _{n \rightarrow \infty} x_{n}$. Now by continuity of $f, x^{*}=\lim _{n \rightarrow \infty} x_{n}$ and $x_{2 n+1}=f\left(x_{2 n}\right)$ imply that $x^{*}$ is a fixed point of $f$. Since $x^{*} \leq x^{*}$, we obtain

$$
f\left(x^{*}\right)-g\left(x^{*}\right) \leq \phi\left(x^{*}-x^{*}\right)
$$

which implies that $x^{*} \in(L F)_{g}$. Now $C F P(f, g, E)$ has a solution as in the above proof. 口

For $f=g$ in Theorem 24, and in Theorem 25 we obtain the following results:

Theorem 26 Let $(E, \leq)$ be a complete vector lattice. Let $f: E \rightarrow E$ be an order continuous and weakly increasing mapping such that for all $x, y \in E$ with $x-y \in K$, we have

$$
f(x)-f(y) \leq \phi(x-y)
$$


where $\phi: K \rightarrow K$ is $(c)$ - comparison operator. If there exists an $x_{0}$ in $(L F)_{f}$ then $F_{f}$ is nonempty.

Theorem 27 Let $(E, \leq)$ be a vector lattice and $K$ a regular cone in $E$. Let $f: K \rightarrow K$ be an order continuous and weakly increasing mappings with respect to the partial order induced by $K$ and

$$
f(x)-f(y) \leq \phi(x-y)
$$

for all $x, y \in K$ with $x-y \in K$ and $\phi: K \rightarrow K$ is (c)-comparison operator. Then the fixed point problem $F P(f, K)$ has a solution.

For $\phi(t)=h t, h \in[0,1), t \in K$ in the above theorems, we obtain the following results:

Theorem 28 Let $(E, \leq)$ be a complete vector lattice. Let $f, g: E \rightarrow E$ be two mutually dominated mappings with respect to $\leq$ and

$$
f(x)-g(y) \leq h(x-y)
$$

provided $x-y \in K$ and $h \in(0,1)$. Then the common fixed point problem $C F P(f, g, E)$ has a solution provided that $f$ is order continuous and $g$ is a dominated map.

Theorem 29 Let $(E, \leq)$ be a vector lattice and $K$ a regular cone. Suppose that $f, g: K \rightarrow$ $K$ are two mutually dominated mappings with respect to order induced by $K$ and

$$
f(x)-g(y) \leq h(x-y)
$$

for all $x, y \in K$ with $x-y \in K$ and $h \in(0,1)$. Then the common fixed point problem $C F P(f, g, K)$ has a solution provided that $f$ is continuous and $g$ is a dominated map.

Theorem 30 Let $(E, \leq)$ be a complete vector lattice. Let $f: E \rightarrow E$ be an order continuous and weakly increasing mapping such that for all $x, y \in E$ with $x-y \in K$, we have

$$
f(x)-f(y) \leq h(x-y)
$$

where $h \in(0,1)$. If there exists an $x_{0}$ in $(L F)_{p}$ then $F_{f}$ is nonempty.

Theorem 31 Let $(E, \leq)$ be a vector lattice and $K$ be a regular cone. Let $f: E \rightarrow E$ bean order continuous and weakly increasing mapping such that for all $x, y \in E$ with $x$ $-y \in K$, we have

$$
f(x)-f(y) \leq h(x-y)
$$

where $h \in(0,1)$. If there exists an $x_{0}$ in $(L F)_{f}$ then $F_{f}$ is nonempty.

Example 32 Let $E=\mathbb{R}_{+}^{2}$ be endowed with the usual ordering in $\mathbb{R}^{2}$ and $f: E \rightarrow E$ be defined by

$$
f\left(x_{2}, x_{1}\right)=\left(\frac{3 x_{1}+2}{5}, \frac{1+4 x_{2}}{5}\right)
$$

For $\left(x_{1}, x_{2}\right),\left(y_{1}, y_{2}\right) \in E$ with $\left(x_{1}, x_{2}\right) \geq\left(y_{1}, y_{2}\right)$, we have

$$
\begin{aligned}
f\left(x_{2}, x_{1}\right)-f\left(y_{1}, y_{2}\right) & =\left(\frac{3 x_{1}+2}{5}, \frac{1+4 x_{2}}{5}\right)-\left(\frac{3 y_{1}+2}{5}, \frac{1+4 y_{2}}{5}\right) \\
& =\left(\frac{3 x_{1}+2}{5}-\frac{3 y_{1}+2}{5}, \frac{1+4 x_{2}}{5}-\frac{1+4 y_{2}}{5}\right) \\
& =\frac{1}{5}\left(3\left(x_{1}-y_{1}\right), 4\left(x_{2}-y_{2}\right)\right) \\
& \leq \frac{1}{5}\left(4\left(x_{1}-y_{1}\right),\left(x_{2}-y_{2}\right)\right) \\
& =\frac{4}{5}\left(\left(x_{1}-y_{1}\right),\left(x_{2}-y_{2}\right)\right) \\
& =\frac{4}{5}\left(\left(x_{1}, x_{2}\right)-\left(y_{1}, y_{2}\right)\right),
\end{aligned}
$$


that is, $f\left(x_{1}, x_{2}\right)-f\left(y_{1}, y_{2}\right) \leq h\left(\left(x_{1}, x_{2}\right)-\left(y_{1}, y_{2}\right)\right)$, where $h=\frac{4}{5} \in(0,1) \in(0,1)$. Moreover, $f$ is a continuous map and $f(1,1)=(1,1)$, that is, $(1,1)$ is a fixed point of $f$. Note that $f(0,0)=\left(\frac{2}{5}, \frac{1}{5}\right)$ and $f(1,1)=(1,1)$. Now $d(f(0,0), f(1,1))=1$ and $d((0,0),(1,1))=$ 1. So the Banach contraction principle is not applicable in this case.

Note that the projection on $K=\mathbb{R}_{+}^{2}$ can easily be obtained here: $P_{K}(x)=P_{k}\left(x_{1}, x_{2}\right)=$ $\left(y_{1}, y_{2}\right)=\left(\max \left\{x_{1}, 0\right\}, \max \left\{x_{2}, 0\right\}\right)$.

As mentioned earlier that solving $N C P(f, K)$ in Hilbert spaces is equivalent to finding a fixed point of the projection mapping given by $F(x)=P_{K}(x-f(x))$. In the following example, $F$ satisfies all the conditions of Theorem 27 and hence has a fixed point.

Example 33 Consider the closed convex cone $K=\mathbb{R}_{+}^{2}$ in the usual $\left(\mathbb{R}^{2}, \leq\right)$. Let $f=\left(f_{1}, f_{2}\right): \mathbb{R}_{+}^{2} \rightarrow \mathbb{R}^{2}$ be defined by

$$
f(x)=\left(f_{1}(x), f_{2}(x)\right)=\left(\frac{x_{1}+1}{2}, \frac{x_{2}-1}{2}\right)
$$

and $F=\left(F_{1}, F_{2}\right): \mathbb{R}^{2} \rightarrow \mathbb{R}_{+}^{2}$ be given by

$$
\begin{aligned}
F(x) & =\left(F_{1}(x), F_{2}(x)\right)=P_{K}\left(\left(x_{1}-f_{1}(x),\left(x_{2}-f_{2}(x)\right)\right)\right. \\
& =\left(\max \left\{\frac{x_{1}-2}{2}, 0\right\}, \max \left\{\frac{x_{2}-1}{2}, 0\right\}\right)
\end{aligned}
$$

Let $x, y \in \mathbb{R}^{2}$ be such that $y=\left(y_{1}, y_{2}\right) \leq x=\left(x_{1}, x_{2}\right)$. Then, $y_{1} \leq x_{1}$ and $y_{2} \leq x_{2}$. If $y_{1} \geq$ 2, then

$$
F_{1}(x)-F_{1}(y)=\frac{x_{1}-2}{2}-\frac{y_{1}-2}{2}=\frac{1}{2}\left(x_{1}-y_{1}\right) .
$$

If $y_{1}<_{2}$, then $x_{1}<_{2}$ gives

$$
F_{1}(x)-F_{1}(y)=0 \leq \frac{1}{2}\left(x_{1}-y_{1}\right) .
$$

If $y_{1}<2$ and $x_{1}>2$, then,

$$
F_{1}(x)-F_{1}(y)=\frac{x_{1}-2}{2}<\frac{x_{1}-2}{2}-\frac{y_{1}-2}{2}=\frac{1}{2}\left(x_{1}-y_{1}\right) .
$$

Consider the case, $y_{2} \leq x_{2}$. If $y_{2} \geq 1$, then

$$
F_{2}(x)-F_{2}(y)=\frac{x_{2}-1}{2}-\frac{y_{2}-1}{2}=\frac{1}{2}\left(x_{2}-y_{2}\right) .
$$

If $y_{2}<1, x_{2}<1$ gives

$$
F_{2}(x)-F_{2}(y)=0 \leq \frac{1}{2}\left(x_{1}-y_{1}\right) .
$$

If $y_{2}<1$ and $x_{2}>1$, then

$$
F_{2}(x)-F_{2}(y)=\frac{x_{2}-1}{2}<\frac{x_{2}-1}{2}-\frac{y_{2}-1}{2}=\frac{1}{2}\left(x_{2}-y_{2}\right) .
$$




$$
F_{2}(x)-F_{2}(y)=0 \leq \frac{1}{2}\left(x_{2}-y_{2}\right)
$$

Thus $F: \mathbb{R}^{2} \rightarrow \mathbb{R}_{+}^{2}$ satisfies all the conditions of Theorem 27. Therefore, $N C P(f, K)$ has a solution.

The following example shows the existence of a solution of the coincidence equation $g(x)=P_{K}(g(x)-f(x))$ which in turn solves $\operatorname{ICP}(f, g, K)$.

Example 34 Consider the closed convex cone $K=\mathbb{R}_{+}^{2}$. Let $f=\left(f_{1}, f_{2}\right): \mathbb{R}_{+}^{2} \rightarrow \mathbb{R}_{+}^{2}$ and $g=\left(g_{1}, g_{2}\right): \mathbb{R}_{+}^{2} \rightarrow \mathbb{R}_{+}^{2}$ be defined by

$$
\begin{aligned}
& f(x)=\left(f_{1}(x), f_{2}(x)\right)=\left(\frac{x_{1}}{2}, \frac{5 x_{2}}{16}\right) \text { and } \\
& g(x)=\left(g_{1}(x), g_{2}(x)\right)=\left(\frac{5 x_{1}}{8}, \frac{5 x_{2}}{8}\right) .
\end{aligned}
$$

Define $F=\left(F_{1}, F_{2}\right): \mathbb{R}_{+}^{2} \rightarrow \mathbb{R}_{+}^{2}$ by

$$
\begin{aligned}
F(x) & =\left(F_{1}(x), F_{2}(x)\right)=P_{K}\left(g_{1}(x)-f_{1}(x), g_{2}(x)-f_{2}(x)\right) \\
& =\left(\frac{x_{1}}{8}, \frac{5 x_{2}}{16}\right) .
\end{aligned}
$$

Let $x, y \in \mathbb{R}^{2}$ such that $y=\left(y_{1}, y_{2}\right) \leq x=\left(x_{1}, x_{2}\right)$. Then, $y_{1} \leq x_{1}$ and $y_{2} \leq x_{2}$.

Now

$$
\begin{aligned}
F_{1}(x)-g_{1}(y) & =\frac{x_{1}}{8}-\frac{5 y_{1}}{8} \\
& \leq \frac{5}{8}\left(x_{1}-y_{1}\right) .
\end{aligned}
$$

If $y_{2}<x_{2}$, then

$$
F_{2}(x)-g_{2}(y)=\frac{5 x_{2}}{16}-\frac{5 y_{2}}{8} \leq \frac{5}{8}\left(x_{2}-y_{2}\right) .
$$

Thus,

$$
F(x)-g(y) \leq \frac{5}{8}(x-y) .
$$

Thus, $F$ and $g$ satisfy all the conditions of Theorem 28. Moreover, $(0,0)$ is a common fixed point of $F$ and $g$ which in turn solves $\operatorname{ICP}(f, g, K)$.

Clearly, a fixed point $p$ of $f$ is also a fixed point of $f^{n}$ for every $n \in \mathbb{N}$. However, the converse is false as follows:

Example 35 Consider, $X=[0,1]$, and define $f$ by $f(x)=1-x^{2}$. Then $f$ has fixed points $\frac{1 \pm \sqrt{5}}{2}$ and $f^{2}$ has fixed points 0 and 1 which are not the fixed points of $f$.

On the other hand, if $X=[0, \pi], f(x)=\cos (x)$, then every iterate of $f$ has the same fixed point as $f$ (cf. [16]).

If a map $T$ satisfies $F_{f}=F_{f^{n}}$ for each $n \in \mathbb{N}$, then it is said to have property $P$ [16]. The set $O(x, \infty)=\left\{x, f(x), f^{2}(x), \ldots\right\}$ is called the orbit of $x$. 
Theorem 36 Let $E, f, K$ and $h$ be as in Theorem 26. If there exists an $x_{0}$ in $U_{f}$ then $f$ has property $P$ provided that $F_{f^{n}} \subseteq(U F)_{f}$.

Proof. The map $f$ has a fixed point by Theorem 26. We shall always assume that $n>$ 1 (since the statement for $n=1$ is trivial). Let $u \in F_{f^{n}}$. Then $f(u) \leq u$ so the nondecreasing characteristic of the mapping $f$ implies that $O(u, \infty)$ is a well ordered subset of $E$. Now we have

$$
\begin{aligned}
u-f(u) & =f\left(f^{n-1}(u)\right)-f^{2}\left(f^{n-1}(u)\right) \leq \phi\left(f^{n-1}(u)-f^{n}(u)\right) \\
& \leq \phi^{2}\left(f^{n-2}(u)-f^{n-1}(u)\right) \leq \ldots \leq \phi^{n}(u-f(u))
\end{aligned}
$$

which, on taking the limit as $n \rightarrow \infty$, implies that $u-f(u) \leq 0$, and hence $u=f(u)$.

It is remarked that, the term "weakly" comes from [15]. Our contractive condition is very much different from the classical one. For example if $K=\mathbb{R}_{+}^{2}$, and for mappings $f$ and $g$ which satisfy $f(x)-f(y)=(-2,-2)$ and $g(x)-g(y)=(1,1)$, then $f(x)-f(y) \leq \frac{1}{2}(g(x)-g(y))$ is true but $|f(x)-f(y)| \leq \frac{1}{2}|(g(x)-g(y))|$ is false.

\section{An application}

Let us consider the implicit integral equation

$$
p(t, x(t))=\int_{0}^{1} q(t, s, x(s)) d s, t, d, s \in[0,1],
$$

where $x \in L^{p}[0,1], 1<p<\infty$. Integral equations like $(I)$ were introduced by Feckan [17] and could occur in the study of nonlinear boundary value problems of ordinary differential equations.

Let $E=L^{p}[0,1]$ be ordered by the following cone:

$$
K=\left\{x \in L^{p}[0,1]: x(t) \leq 0, \text { almost every where }\right\} .
$$

We assume the following:

(1) For all $x, y \in E$ with $x-y \in K$,

$$
p(t, x(t))-p(t, y(t)) \leq h(x(t)-\gamma(t)),
$$

where $t \in[0,1]$.

(2) $q(t, s, x(s)) \leq x(t)$ for all $t \in[0,1]$

(3) $p\left(t, \int_{0}^{1} q(t, s, x(s)) d s\right) \leq \int_{0}^{1} q(t, s, x(s)) d s$ for all $t, s \in[0,1]$.

(4) $q(t, s, x(s)) \geq p(t, x(t))$ for all $t, s \in[0,1]$.

Then, the implicit integral equation (I) has a solution in $L^{p}[0,1]$.

Proof. Define

$$
f(x)(t)=p(t, x(t)) \text { and } g(x)(t)=\int_{0}^{1} q(t, s, x(s)) d s
$$


By using (3), we have

$$
g(x)(t)=\int_{0}^{1} q(t, s, x(s)) d s \leq \int_{0}^{1} x(t) d s=x(t)
$$

which implies that $x(t)-g(x)(t) \in K$ and hence $g$ is dominated. Further, using (3) and (4), we obtain

$$
f(g(x))(t)=p\left(t, \int_{0}^{1} q(t, s, x(s)) d s\right) \leq \int_{0}^{1} q(t, s, x(s)) d s=g(x)(t)
$$

and

$$
g(f(x))(t)=\int_{0}^{1} q(t, s, p(t, x(s))) d s \leq p(t, x(s))=f(x)(t) .
$$

So, $f$ and $g$ are mutually dominated mappings. Finally, we have

$$
\begin{aligned}
f(x)(t)-g(\gamma)(t) & =p(t, x(t))-\int_{0}^{1} q(t, s, y(s)) d s \\
& \leq p(t, x(t))-\int_{0}^{1} p(t, \gamma(t)) d s \\
& =p(t, x(t))-p(t, y(t)) \\
& \leq h(x(t)-\gamma(t)),
\end{aligned}
$$

that is, $f(x)(t)-g(y)(t) \leq h(x(t)-y(t))$ is satisfied for all $t \in[0,1]$. By using Theorem 29, the common fixed point problem $\operatorname{CFP}(f, g, K)$ has a solution which in turn solves the integral equation (I).

\section{Acknowledgements}

The authors are thankful to the anonymous referees for their critical remarks which helped to improve the presentation of the paper. A. R. Khan is grateful to King Fahd University of Petroleum and Minerals for supporting the research project IN101037. M. Abbas was supported by Higher Education Commission, Pakistan.

\section{Author details}

${ }^{1}$ Lahore University of Management Sciences (LUMS), 54792 Lahore, Pakistan ${ }^{2}$ Department of Mathematics and Statistics, King Fahd University of Petroleum and Minerals, Dhahran 31261, Saudi Arabia ${ }^{3}$ School of Mathematics, The University of Birmingham, The Watson Building, Edgbaston, Birmingham B15 2TT, UK

\section{Authors' contributions}

The authors have contributed in this work on an equal basis.All authors read and approved the final manuscript.

\section{Competing interests}

The authors declare that they have no competing interests.

Received: 8 September 2011 Accepted: 16 April 2012 Published: 16 April 2012

\section{References}

1. Amman, H: Order structures and fixed points. Math Instrum Ruhr. (1997)

2. Cristescu, R: Order Vector Spaces and Linear Operators. Academiei-Abacus Press Kent (1976)

3. Heikkila, S: On fixed points through iteratively generated chains with applications to differential equations. J Math Anal Appl. 138, 397-417 (1989)

4. Hussain, N, Khan, AR, Agarwal, RP: Krasnoselskii and Ky Fan-type fixed point theorems in ordered Banach spaces. J Nonlinear Convex Anal. 11, 475-489 (2010)

5. Berinde, V: Generalized contractions in $\sigma$-complete vector lattices. Univ u Novom Sadu Zb Rad Prirod-Mat Fak Ser Mat. 24(2):31-38 (1994)

6. Isac, G, Németh, AB: Projection methods, isotone projection cones, and the complementarity problem. J Math Anal Appl. 153, 258-275 (1990)

7. Isac, G: Problem de complementarite (En dimention infinte). Minicourse Publication Depart Math Univ. Limoges France (1985) 
8. Isac, G: Complementarity problem and coincidence equation on convex cones. Boll Un Mat Ital. 5-B(6):925-943 (1986)

9. Ran, $A C M$, Reurings, $M C B$ : A fixed point theorem in partially ordered sets and some applications to matrix equations. Proc Am Math Soc. 132, 1435-1443 (2004)

10. Nieto, JJ, Lopez, RR: Contractive mapping theorems in partially ordered sets and applications to ordinary differential equations. Order. 22, 223-239 (2005)

11. Abbas, M, Khamsi, MA, Khan, AR: Common fixed point and invariant approximation in hyperbolic ordered metric spaces. Fixed Point Theory Appl. 2011, 25 (2011)

12. Amini-Harandi, A, Emami, H: A fixed point theorem for contraction type maps in partially ordered metric spaces and application to ordinary differential equations. Nonlinear Anal. 72, 2238-2242 (2010)

13. Ciric, L, Abbas, M, Saadati, R, Hussain, N: Common fixed points of almost generalized contractive mappings in ordered metric spaces. Appl Math Comput. 217, 5784-5789 (2011)

14. Harjani, J, Sadarangani, K: Fixed point theorems for weakly contractive mappings in partially ordered sets. Nonlinear Anal. 71, 3403-3410 (2009)

15. Nemeth, SZ: Iterative methods for nonlinear complementarity problems on isotone projction cones. Math Anal Appl. 350, 340-347 (2009)

16. Jeong, GS, Rhoades, BE: Maps for which $F(T)=F\left(T^{n}\right)$. Fixed Point Theory Appl. 6, 71-105 (2007)

17. Fečkan, M: Nonnegtive solutions of nonlinear integral equations. Comments Math Univ Carolinae. 36, 615-627 (1995)

doi:10.1186/1687-1812-2012-60

Cite this article as: Abbas et al: Complementarity problems via common fixed points in vector lattices. Fixed Point Theory and Applications 2012 2012:60.

\section{Submit your manuscript to a SpringerOpen ${ }^{\circ}$} journal and benefit from:

- Convenient online submission

- Rigorous peer review

- Immediate publication on acceptance

- Open access: articles freely available online

- High visibility within the field

- Retaining the copyright to your article

Submit your next manuscript at $\boldsymbol{s p r i n g e r o p e n . c o m ~}$ 